This is an Accepted Manuscript of an article published by Portland Press.

Biochemical Society Transactions. 2017.45(2):475-464. Available online: http://

dx.doi.org/10.1042/BST20160272.

\title{
Targeting ROR1 identifies new treatment strategies in hematological cancers
}

\author{
Hanna Karvonen ${ }^{1}$, Wilhelmiina Niininen ${ }^{1}$, Astrid Murumägi ${ }^{2}$ and Daniela Ungureanu ${ }^{1}$ \\ ${ }^{1}$ BioMediTech, BMT, University of Tampere, 33014, Tampere, Finland \\ ${ }^{2}$ Institute for Molecular Medicine Finland, FIMM, University of Helsinki, 00290, Helsinki, Finland
}

Corresponding author name and email:

Daniela Ungureanu

email: daniela.ungureanu@uta.fi

Abbreviations: ALL, acute lymphocytic leukemia; B-ALL, B-cell acute lymphoblastic leukemia; BCR, Bcell receptor; BTK, Bruton tyrosine kinase; CLL, chronic lymphocytic leukemia; mAb, monoclonal antibody; MCL, mantle cell lymphoma; NHL, Non-Hodgkin lymphoma; ROR1/2, Receptor tyrosine kinase-like orphan receptor $1 / 2$; Wnt, wingless-related integration site

\begin{abstract}
ROR1 is a member of the ROR receptor family consisting of two closely related type I transmembrane proteins, ROR1 and ROR2. Due to mutations in their canonical motifs required for proper kinase activity, RORs are classified as pseudokinases lacking detectable catalytic activity. ROR1 stands out for its selective and high expression in numerous blood and solid malignancies compared to a minimal expression in healthy adult tissues, suggesting high potential for this molecule as drug target for cancer therapy. Current understanding attributes a survival role for ROR1 in cancer cells, however its oncogenic function is cancer-type specific and involves various signaling pathways. High interest in ROR1 targeted therapies resulted in the development of ROR1 monoclonal antibodies such as
\end{abstract}


cirmtuzumab, currently in phase I clinical trial for CLL. Despite these advances in translational studies, the molecular mechanism employed by ROR1 in different cancers is not yet fully understood, therefore more insights into the oncogenic role of ROR1 signaling are crucial in order to optimize the use of targeted drugs. Recent studies provided evidence that targeting ROR1 simultaneously with inhibition of BCR signaling is more effective in killing ROR1-positive leukemia cells, suggesting a synergistic correlation between co-targeting ROR1 and BCR pathways. Although this synergy has been previously reported for B-ALL, the molecular mechanism appears rather different. These results provide more insights into ROR1-BCR combinatorial treatment strategies in hematological malignancies, which could benefit in tailoring more effective targeted therapies in other ROR1-positive cancers.

ROR family of pseudokinases

ROR family of proteins belongs to the non-canonical Wnt signaling pathway and comprises of two type I, single-pass transmembrane glycoproteins, ROR1 and ROR2, closely related to MuSK (muscle-specific kinase) and Trk (tropomyosin) family receptors [1, 2].

The extracellular region (ectodomain) contains an immunoglobulin-like domain (IgG), a cysteine-rich domain (CRD) with sequence and structural similarities to the CRD of Frizzled proteins followed by a Kringle domain. A single transmembrane helix connects the ectodomain to the cytoplasmic region, which contains a tyrosine kinase-like or pseudokinase domain followed by two serine/threonine (Ser/Thr) rich domains flanking a proline (Pro) rich domain (Figure 1A). ROR ligands were initially unknown, hence their "orphan" denomination, however subsequent studies have demonstrated that both ROR1 and to a greater extent ROR2 are able to bind Wnt5a presumably via their extracellular CRD domain [3-7]. ROR Kringle domain, a highly-folded cysteine-rich domain that mediates protein-ligand interaction in coagulation proteins, appears to play a role in ROR1/ROR2 heterodimerization and subsequent activation of Rho/Rac1 GTPase pathway in leukemia cells [7]. The cytoplasmic tyrosine kinase-like domain lacks several conserved residues important for phosphotransferase activity, therefore RORs are classified as pseudokinases (Figure 1B). Although early studies reported evidence of autocatalytic activity for both ROR1 and ROR2 $[1,8,9]$, the prevailing view of RORs is as inactive pseudokinases, since using purified recombinant proteins the nucleotide binding and autocatalytic activity could not be detected for either of $\operatorname{ROR}[10,11]$. X-ray crystallographic studies of unphosphorylated ROR2 pseudokinase domain revealed an activation loop that occludes the substrate- and ATP- binding sites via the unique position of Tyr555 side chain, a feature not observed 
in other kinases [12]. Sequence alignment of ROR1 and ROR2 pseudokinase domains shows high-level of homology (Figure 1B), indicative of similar functional mechanisms. Interestingly, restoration of glycine-rich loop to its canonical consensus sequence does not result in an increased in vitro catalytic activity for purified ROR cytoplasmic domains, suggesting a predominantly non-catalytic function for these pseudokinases [10]. Functional evidence for ROR1 catalytic activity came from studies using mutation of the invariant Lys506 in $\beta 3$-sheet to create a kinase dead enzyme. ROR1 K506A mutant could not sustain downstream signaling and growth advantage in lung adenocarcinoma cells suggesting that ROR1 kinase activity is required for cell survival [13]. Similar studies have shown that ROR1 could trans-phosphorylate HER3 at a previously unidentified Tyr1307 site and this phosphorylation was abolished by using ROR1 K506A kinase dead mutant [14]. While K506A mutation may alter ROR1 structural integrity and compromise its downstream signaling without affecting its socalled kinase activity, the possibility of a very low or atypical enzymatic activity could exist. The insolubility of both RORs K506R mutants as recombinant proteins (cytoplasmic domain) compared to fully soluble wild-type proteins leave us unable to provide a clear biochemical evidence for the effect of this mutation on ROR catalytic properties in vitro [10]. Phosphorylation of ROR1 has been observed in cell lysates by using either ROR1 phospho-specific or pan-phosphotyrosine antibodies, and several kinases including Src and Met were shown to directly interact with and phosphorylate ROR1, demonstrating that ROR1 takes part in the dynamic network of intracellular phosphorylation events either as a substrate or presumably as an active kinase $[13,15-17]$. While ROR1/ROR2 heterodimerization occurs in response to Wnt5a binding, ligand-induced ROR1/ROR2 transphosphorylation or auto-phosphorylation have not been detected thus far [7, 10]. The ability of ROR1 to phosphorylate exogenous substrates needs to be investigated beyond K506A mutant overexpression, ruling out the possibility that intermolecular interactions could be responsible for the observed functional effect.

The Pro-rich domain of ROR1 serves as substrate for Met phosphorylation in cells with Metamplification and as a binding site for Src kinase, while recent reports have added new functions for this domain as binding site for 14-3-3 3 and HS1 (hematopoietic cell-specific Lyn substrate 1) to mediate downstream signaling leading to cell proliferation and leukemia cell migration $[13,15,16,18,19]$. On the other hand, ROR2 Pro-rich domain serves as a binding site and substrate for CKIE kinase resulting in serine-threonine phosphorylation of the second Ser/Thr domain followed by subsequent tyrosine phosphorylation of ROR2 pseudokinase domain [3]. The functional role of the C-terminal Ser/Thr domains in ROR1 awaits further studies. 
ROR family of proteins are evolutionally conserved and share relatively high level of homology from Drosophila to mammals, underlining their important biological functions [20]. In line with this, mROR1 ${ }^{-/}$mice show postnatal growth retardation and have a reduced life expectancy, whereas mROR2 ${ }^{-/}$mice die at birth due to severe skeletal and heart abnormalities [21-23]. mROR1/mROR2deficient mouse showed a more severe phenotype than mROR2 ${ }^{-/}$alone, indicating that mROR1 and mROR2 are functionally redundant during embryonic development [24] .

The strong ROR1 expression during normal embryonic and fetal development is not sustained in all postpartum healthy tissues. Low ROR1 levels were detected in adipose cells and to a lesser degree in the pancreas, lung and a subset of intermediate B cells [25-27]. Moreover, using a more sensitive ROR1-specific monoclonal antibody recent studies have shown relatively high expression levels of ROR1 in parathyroid, pancreatic islets and multiple regions in the gut [28]. However, upregulation of ROR1 is commonly detected in both hematological and solid tumors [29], which attracted high scientific interest to explore the functional advantage of targeting ROR1 expression in cancer cells as a therapeutic strategy.

ROR1 and hematological cancers

ROR1 overexpression was initially identified on B-cells from chronic lymphocytic leukemia (CLL) but not on normal B-cells from healthy donors, suggesting that ROR1 expression could become a marker for CLL [25]. Furthermore, several research groups have identified ROR1 but not ROR2 expression in many other hematological malignancies, indicating that ROR1 is more prevalent in blood cancers. ROR1 upregulation was detected in primary samples of mantle cell lymphoma (MCL), marginal zone lymphoma (MZL), diffuse large B-cell lymphoma (DLBCL) and follicular lymphoma (FL), and high levels of ROR1 expression is seen in B-ALL patients with the $t(1 ; 19)(q 23 ; p 13)$ translocation $[27,30,31]$. It is now generally acknowledged that ROR1 expression is commonly detected in NHLs, ranging from high levels in CLL to moderate levels in MCL or MZL, suggesting that ROR1 could play a role in the induction and/or maintenance of B-cell lymphomagenesis.

A careful assessment of ROR1 expression on normal and leukemic B cells at different stages of B-cell development has helped us to understand how malignant B cells acquired ROR1 for their survival advantage. ROR1 expression is completely absent at early stages of normal B-cell development, becomes highly expressed at intermediate/late stages (on precursor B cells called hematogones), and is then downregulated in normal, mature B cells. On the other hand, ROR1 expression was observed in 
both, intermediate and mature B-cell malignancies suggesting that retention of ROR1 expression beyond the intermediate stage could be an important survival step for these mature, malignant B cells $[26,27,32]$. Supporting this idea, recent transcriptome analysis of CLL patient samples showed that CLL cells with higher ROR1 expression levels have enhanced activation of AKT signaling compared to CLL cells with lower ROR1 expression, and this correlated with enhanced disease progression and shorter survival rate [33]. Similarly, ROR1 expression in t(1;19) B-ALL cells could sustain pro-survival signaling through activation of MEK/ERK and AKT pathways [27]. These pathways control numerous cellular responses, such as proliferation, cell cycle entry, survival, metabolic pathway activation, apoptosis and angiogenesis. Interestingly, low ROR2 expression was found on both, normal $\mathrm{CD}^{+} \mathrm{B}^{-}$ cells as well as malignant B cells isolated from CLL patients. ROR1/ROR2 heterodimerization is required for Wnt5a-mediated chemotaxis and proliferation of leukemic CLL cells [7]. Apart from ROR1 and ROR2, Wnt5a binds to RYK (receptor tyrosine kinase) and PTK7 (protein tyrosine kinase 7), two other pseudokinases from the RTK family [34]. Wnt5a plays a variety of roles in different types of tumors, and the complexity of its signaling pathways depends largely on the receptors expression, downstream effectors, exogenous inhibitors and tumor microenvironments, as well as the existing cross-talk with the canonical Wnt-pathways [35]. It remains to be investigated whether the non-canonical Wnt5aROR1/ROR2 pathway is functional in other ROR-positive hematological cancers.

\section{ROR1 targeted therapies}

Given the restricted overexpression of ROR1 on tumor cells and not on most healthy adult tissues, several studies have started to explore ROR1 targeted therapies. Two major pharmacological strategies were developed: monoclonal antibodies ( $\mathrm{mAb}$ ) targeting the extracellular domain of the receptor, and low molecular weight compounds that target the intracellular domain. Silencing ROR1 expression with siRNA resulted in efficient apoptosis of leukemic cells, and the same cytotoxic effect was observed by targeting ROR1 with mAb $[36,37]$. Several ROR1 mAbs have been developed and their cytotoxic properties depended largely on their distinctive epitopes. ROR1 mAbs targeting the CRD or Kringle domain were more effective in directly killing CLL cells than CD20 mAb rituximab, currently approved for advanced CLL treatment, whereas antibodies directed against IgG-like domain were less cytotoxic in directly mediating apoptotic effects $[37,38]$. Humanized ROR1 mAb cirmtuzumab or UC-961 targeting an epitope at the junction of IgG-like and CRD domain demonstrated specific and high cytotoxic effect against ROR1-positive leukemic cells in vitro and in vivo, and currently advanced to phase I clinical trial for CLL [39]. The preclinical efficacy of UC-961 in other ROR1-positive cancers 
including solid tumors such as ovarian cancer indicated that this mAb could have broader therapeutic application. More studies will be required to fully characterize the direct or indirect effector mechanisms of different ROR1 mAbs and their potential interference with yet unknown receptor/ligand interactions leading to undesired agonistic activity. On the other hand, targeting the intracellular domain of ROR1 by small molecule inhibitor such as KAN0439834 demonstrated promising preclinical efficiency by inducing high levels of apoptosis/necrosis in CLL cells, indicating that ROR1 could become a druggable therapeutic target [40].

A better understanding of the signaling pathway downstream ROR1 has been derived from recent studies using mass-spectrometry analysis of ROR1 immunoprecipitated samples from CLL cells. Wnt5a binding can induce ROR1/ROR2 dimerization and enhance cell proliferation and/or leukemia-cell migration. ROR1 mAb UC-961 was shown to compete with Wnt5a and abrogate the heterodimerization of ROR1/ROR2 and subsequent intracellular signaling cascade employing guanine exchange factors (GEFs) and their downstream effectors Rho and Rac1 resulting in inhibition of cell proliferation and chemokine-induced migration [7]. Though most of the attention has been focused on ROR1 signaling in CLL models, a deeper understanding of ROR1 molecular pathways in other hematological cancers will help in designing more effective targeted therapies.

\section{Co-targeting BCR and ROR1 in B-cell malignancies}

Mature B lymphocytes are essential antibody-producing cells of the immune system harboring B-cell receptors that recognize foreign antigens. Signals via the BCRs are responsible for development/maturation, proliferation, maintenance, and activation of normal B lymphocytes and proceed through several downstream BCR-related kinases [41]. These signaling events are not only prevalent but also overexpressed in malignant B lymphocytes, and targeting various components of this pathway appear to be the most promising therapy. As such, inhibition of BCR-downstream effector kinases demonstrated high therapeutic efficacy as evidenced by past and ongoing clinical trials for BTK inhibitor ibrutinib, Syk inhibitor fostamatinib, and PI3K $\delta$ inhibitor idelalisib [42-45]. Considerable attention has been given to ibrutinib for its unprecedented clinical efficacy as a single agent in CLL and MCL patients [46]. However, tumor relapse is still a challenging fate for many patients, indicating that combinatorial treatment strategies must be considered.

Although ROR1 and BCR signaling pathways are driven by the engagement of unrelated cell-surface receptors, a regulatory feedback loop via common downstream mediators has been identified in B-ALL 
cells [27]. Silencing of pre-BCR expression in $t(1 ; 19)$ B-ALL cells resulted in significant up-regulation of ROR1 protein levels, suggesting an intrinsic regulatory mechanism that function via PI3K/AKT pathway to maintain cell growth and viability. Pre-BCR silencing induced a positive feedback loop for upregulation of ROR1 expression, which in turn drives reactivation of AKT signaling resulting in increased cell-survival. As such, simultaneous ROR1 knockdown and pre-BCR inhibition is more effective in killing t(1;19)B-ALL cells than targeting either pathway alone, as demonstrated by the effectiveness of dasatinib treatment (a multi-kinase inhibitor targeting BTK and Src kinases downstream BCR pathway) together with siRNA-mediated ROR1 silencing (Figure 2).

A similar synergism has been recently described for leukemic CLL cells, where co-targeting ROR1 and BTK by cirmtuzumab and ibrutinib co-treatment is more effective in killing leukemia cells in vitro and in vivo than either agent alone [47] (Figure 2). Wnt5a-induced Rac1 activation could be inhibited by cirmtuzumab treatment but not by ibrutinib-mediated BTK-inhibition, suggesting that ROR1 and BCR signaling pathways are independently modulated in CLL cells. The synergistic effect of cirmtuzumab and ibrutinib could provide a solution for already challenging ibrutinib acquired resistance, as it occurs in many patients undergoing ibrutinib monotherapy.

Combination therapy is now widely considered as the optimal strategy especially for overcoming drugresistance more permanently. Therefore, a significant amount of research is currently focused on identifying and testing rational combinatorial treatments. Co-targeting BCR and non-canonical Wnt signaling pathways in blood cancers is an area less investigated thus far. The functional link between ROR1 and pre-BCR signaling converging downstream at the level of AKT/PI3K phosphorylation in B-ALL cells indicates that common signaling mediators can significantly modulate the outcome of combinatorial therapies. While is unknown whether ROR1 signaling in B-ALL requires ligand-mediated activation, in CLL cells Wnt5a binding can increase motility and chemotactic responses of ROR1 expressing cells. Inhibition of ROR1 signaling by cirmtuzumab resulted in decreased cell proliferation and chemokine-directed migration, indicating that Wnt5a-ROR1 signaling is involved in leukemia cell chemotaxis, a function not related to ROR1 kinase activity [7]. Several BCR effector kinases such as BTK, PI3K and Syk play important roles in pathways activated by tumor-microenvironment interactions, therefore BCR targeted inhibitors are known to interfere with chemokine-mediated migration of CLL cells, impending the ability of cancer cells to "home" to the microenvironment $[45,48,49]$. As such, we could hypothesize that co-targeting ROR1 and BCR signaling will trigger this common effector mechanism and will result in augmented inhibition of chemokine-mediated migration of leukemic cells and their survival signals from tumor microenvironment. Targeting ROR1 offers the advantage of low 
cytotoxicity due to its restricted expression in healthy adult tissue, therefore the additional toxicity that may be expected with combination therapy could be significantly minimized. Interestingly, another Wnt5a binding and catalytically inactive pseudokinase PTK7 was found to be expressed in AML (acute myeloid leukemia) and its expression correlated with increased leukemic cell migration, survival and resistance to anthracycline-induced apoptosis $[11,50]$. This could indicate that chemotaxis is a common mechanism mediated by non-canonical Wnt receptor pseudokinases in leukemic cells.

The complexity of BCR signaling regulation in various NHLs is directly reflected by different sensitivities of BCR targeted drugs for each disease model. Therefore, some drug combinations could synergize better in a particular disease type but not in others. Consequently, a combination therapy by cotargeting ROR1 and BCR signaling should be carefully investigated for each ROR1-positive lymphoma entities in order to identify more specific and effective combinatorial treatments.

\section{Conclusion}

Past and ongoing clinical trials with targeted drugs in cancer have shown us that single drugs are rarely effective in achieving long-term remission due to existing or acquired compensatory pathways. From this perspective, effective cancer treatments will only come from the rational combination of targeted agents, derived from a deep knowledge of signaling pathways and their interconnectedness. While currently is difficult to predict the true clinical potential of ROR1 targeted therapy (either by mAbs or small molecule inhibitors), results obtained thus far could provide a glimpse of the future. Therefore, it seems safe predicting that anti-ROR1 agents, especially mAbs, will likely be combined with other targeted drugs in order to achieve the best clinical outcome. Taken together, from the perspective of therapeutic efficacy, these preclinical observations support the concept that a combination therapy with ROR1 and BCR targeted agents for ROR1-positive B-cell malignancies will likely be clinically advantageous.

\section{Declaration of interest}

The authors declare no conflict of interest.

\section{Funding}


This work was supported by the Medical Research Council of Academy of Finland and Doctoral Programme in Biomedicine and Biotechnology at University of Tampere, Finland.

\section{References}

1. Masiakowski P, Carroll RD (1992) A novel family of cell surface receptors with tyrosine kinaselike domain. J Biol Chem 267:26181-26190

2. Forrester WC, Dell M, Perens E, Garriga G (1999) A C. elegans Ror receptor tyrosine kinase regulates cell motility and asymmetric cell division. Nature 400:881-885

3. Oishi I, Suzuki H, Onishi N, et al (2003) The receptor tyrosine kinase Ror2 is involved in noncanonical Wnt5a/JNK signalling pathway. Genes Cells Devoted Mol Cell Mech 8:645-654

4. Mikels AJ, Nusse R (2006) Purified Wnt5a protein activates or inhibits beta-catenin-TCF signaling depending on receptor context. PLoS Biol 4:e115

5. Fukuda T, Chen L, Endo T, et al (2008) Antisera induced by infusions of autologous Ad-CD154leukemia B cells identify ROR1 as an oncofetal antigen and receptor for Wnt5a. Proc Natl Acad Sci U S A 105:3047-3052

6. Paganoni S, Bernstein J, Ferreira A (2010) Ror1-Ror2 complexes modulate synapse formation in hippocampal neurons. Neuroscience 165:1261-1274

7. Yu J, Chen L, Cui B, Widhopf GF, Shen Z, Wu R, Zhang L, Zhang S, Briggs SP, Kipps TJ (2016) Wnt5a induces ROR1/ROR2 heterooligomerization to enhance leukemia chemotaxis and proliferation. J Clin Invest 126:585-598

8. Oishi I, Takeuchi S, Hashimoto R, et al (1999) Spatio-temporally regulated expression of receptor tyrosine kinases, mRor1, mRor2, during mouse development: implications in development and function of the nervous system. Genes Cells Devoted Mol Cell Mech 4:41-56

9. Liu Y, Rubin B, Bodine PVN, Billiard J (2008) Wnt5a induces homodimerization and activation of Ror2 receptor tyrosine kinase. J Cell Biochem 105:497-502

10. Bainbridge TW, DeAlmeida VI, Izrael-Tomasevic A, et al (2014) Evolutionary divergence in the catalytic activity of the CAM-1, ROR1 and ROR2 kinase domains. PloS One 9:e102695

11. Murphy JM, Zhang Q, Young SN, et al (2014) A robust methodology to subclassify pseudokinases based on their nucleotide-binding properties. Biochem J 457:323-334

12. Artim SC, Mendrola JM, Lemmon MA (2012) Assessing the range of kinase autoinhibition mechanisms in the insulin receptor family. Biochem J 448:213-220

13. Yamaguchi T, Yanagisawa K, Sugiyama R, et al (2012) NKX2-1/TITF1/TTF-1-Induced ROR1 is required to sustain EGFR survival signaling in lung adenocarcinoma. Cancer Cell 21:348-361 
14. Li C, Wang S, Xing Z, et al (2017) A ROR1-HER3-IncRNA signalling axis modulates the Hippo-YAP pathway to regulate bone metastasis. Nat Cell Biol 19:106-119

15. Gentile A, Lazzari L, Benvenuti S, Trusolino L, Comoglio PM (2011) Ror1 is a pseudokinase that is crucial for Met-driven tumorigenesis. Cancer Res 71:3132-3141

16. Gentile A, Lazzari L, Benvenuti S, Trusolino L, Comoglio PM (2014) The ROR1 pseudokinase diversifies signaling outputs in MET-addicted cancer cells. Int J Cancer J Int Cancer 135:23052316

17. Hojjat-Farsangi M, Khan AS, Daneshmanesh AH, Moshfegh A, Sandin $\AA$, Mansouri L, Palma M, Lundin J, Österborg A, Mellstedt H (2013) The Tyrosine Kinase Receptor ROR1 Is Constitutively Phosphorylated in Chronic Lymphocytic Leukemia (CLL) Cells. PLOS ONE. doi: 10.1371/journal.pone.0078339

18. Yu J, Chen L, Chen Y, Zhang L, Rassenti LZ, Widhopf GF, Shen Z, Briggs SP, Kipps TJ (2016) Wnt5a Induces Association of ROR1 with 14-3-3ろ to Enhance Chemotaxis and Proliferation in Chronic Lymphocytic Leukemia. Blood 128:349-349

19. Hasan MK, Yu J, Chen L, Cui B, Shen Z, Widhopf GF, Rassenti LZ, Briggs SP, Kipps TJ (2016) Wnt5a Induces ROR1 to Complex with HS1, Which Undergoes Tyrosine Phosphorylation and Contributes to Planar-Cell-Polarity Migration in Chronic Lymphocytic Leukemia. Blood 128:301301

20. Yoda A, Oishi I, Minami Y (2003) Expression and Function of the Ror-Family Receptor Tyrosine Kinases During Development: Lessons from Genetic Analyses of Nematodes, Mice, and Humans. J Recept Signal Transduct 23:1-15

21. Lyashenko N, Weissenböck M, Sharir A, Erben RG, Minami Y, Hartmann C (2010) Mice lacking the orphan receptor ror1 have distinct skeletal abnormalities and are growth retarded. Dev Dyn 239:2266-2277

22. Takeuchi S, Takeda K, Oishi I, et al (2000) Mouse Ror2 receptor tyrosine kinase is required for the heart development and limb formation. Genes Cells Devoted Mol Cell Mech 5:71-78

23. Schwabe GC, Trepczik B, Süring K, Brieske N, Tucker AS, Sharpe PT, Minami Y, Mundlos S (2004) Ror2 knockout mouse as a model for the developmental pathology of autosomal recessive Robinow syndrome. Dev Dyn Off Publ Am Assoc Anat 229:400-410

24. Nomi M, Oishi I, Kani S, et al (2001) Loss of mRor1 enhances the heart and skeletal abnormalities in mRor2-deficient mice: redundant and pleiotropic functions of mRor1 and mRor2 receptor tyrosine kinases. Mol Cell Biol 21:8329-8335

25. Baskar S, Kwong KY, Hofer T, Levy JM, Kennedy MG, Lee E, Staudt LM, Wilson WH, Wiestner A, Rader C (2008) Unique Cell Surface Expression of Receptor Tyrosine Kinase ROR1 in Human BCell Chronic Lymphocytic Leukemia. Clin Cancer Res 14:396-404

26. Hudecek M, Schmitt TM, Baskar S, et al (2010) The B-cell tumor-associated antigen ROR1 can be targeted with T cells modified to express a ROR1-specific chimeric antigen receptor. Blood 116:4532-4541 
27. Bicocca VT, Chang BH, Masouleh BK, Muschen M, Loriaux MM, Druker BJ, Tyner JW (2012) Crosstalk between ROR1 and the Pre-B cell receptor promotes survival of $t(1 ; 19)$ acute lymphoblastic leukemia. Cancer Cell 22:656-667

28. Balakrishnan A, Goodpaster T, Randolph-Habecker J, et al (2016) Analysis of ROR1 protein expression in human cancer and normal tissues. Clin Cancer Res Off J Am Assoc Cancer Res. doi: 10.1158/1078-0432.CCR-16-2083

29. Borcherding N, Kusner D, Liu G-H, Zhang W (2014) ROR1, an embryonic protein with an emerging role in cancer biology. Protein Cell 5:496-502

30. Barna G, Mihalik R, Timár B, et al (2011) ROR1 expression is not a unique marker of CLL. Hematol Oncol 29:17-21

31. Daneshmanesh AH, Porwit A, Hojjat-Farsangi M, et al (2013) Orphan receptor tyrosine kinases ROR1 and ROR2 in hematological malignancies. Leuk Lymphoma 54:843-850

32. Broome HE, Rassenti LZ, Wang H-Y, Meyer LM, Kipps TJ (2011) ROR1 is expressed on hematogones (non-neoplastic human B-lymphocyte precursors) and a minority of precursor-B acute lymphoblastic leukemia. Leuk Res 35:1390-1394

33. Cui B, Ghia EM, Chen L, et al (2016) High-level ROR1 associates with accelerated disease progression in chronic lymphocytic leukemia. Blood 128:2931-2940

34. Niehrs C (2012) The complex world of WNT receptor signalling. Nat Rev Mol Cell Biol 13:767779

35. Asem MS, Buechler S, Wates RB, Miller DL, Stack MS (2016) Wnt5a Signaling in Cancer. Cancers. doi: 10.3390/cancers8090079

36. Choudhury A, Derkow K, Daneshmanesh AH, Mikaelsson E, Kiaii S, Kokhaei P, Osterborg A, Mellstedt $H$ (2010) Silencing of ROR1 and FMOD with siRNA results in apoptosis of CLL cells. Br J Haematol 151:327-335

37. Daneshmanesh AH, Hojjat-Farsangi M, Khan AS, et al (2012) Monoclonal antibodies against ROR1 induce apoptosis of chronic lymphocytic leukemia (CLL) cells. Leukemia 26:1348-1355

38. Baskar S, Wiestner A, Wilson WH, Pastan I, Rader C (2012) Targeting malignant B cells with an immunotoxin against ROR1. mAbs 4:349-361

39. Choi MY, Widhopf GF, Wu CCN, et al (2015) Pre-clinical Specificity and Safety of UC-961, a FirstIn-Class Monoclonal Antibody Targeting ROR1. Clin Lymphoma Myeloma Leuk 15:S167-S169

40. Hojjat-Farsangi M, Moshfegh A, Daneshmanesh AH, et al (2015) First-in-Class ROR1 Small Molecule Inhibitor (KAN0439834) Downregulated Wnt-Canonical and Non-Canonical Signaling Pathways and Induced Apoptosis of CLL Cells. Blood 126:2912-2912

41. Rickert RC (2013) New insights into pre-BCR and BCR signalling with relevance to $B$ cell malignancies. Nat Rev Immunol 13:578-591 
42. Herman SEM, Barr PM, McAuley EM, Liu D, Wiestner A, Friedberg JW (2013) Fostamatinib inhibits B-cell receptor signaling, cellular activation and tumor proliferation in patients with relapsed and refractory chronic lymphocytic leukemia. Leukemia 27:1769-1773

43. Byrd JC, Furman RR, Coutre SE, et al (2013) Targeting BTK with ibrutinib in relapsed chronic lymphocytic leukemia. N Engl J Med 369:32-42

44. Cheng S, Ma J, Guo A, Lu P, Leonard JP, Coleman M, Liu M, Buggy JJ, Furman RR, Wang YL (2014) BTK inhibition targets in vivo CLL proliferation through its effects on B-cell receptor signaling activity. Leukemia 28:649-657

45. Hoellenriegel J, Meadows SA, Sivina M, et al (2011) The phosphoinositide 3'-kinase delta inhibitor, CAL-101, inhibits B-cell receptor signaling and chemokine networks in chronic lymphocytic leukemia. Blood 118:3603-3612

46. McDermott J, Jimeno A (2014) Ibrutinib for the treatment of chronic lymphocytic leukemia and mantle cell lymphoma. Drugs Today Barc Spain 1998 50:291-300

47. Yu J, Chen L, Cui B, Wu C, Choi MY, Chen Y, Zhang L, Rassenti LZ, Widhopf li GF, Kipps TJ (2017) Cirmtuzumab inhibits Wnt5a-induced Rac1 activation in chronic lymphocytic leukemia treated with ibrutinib. Leukemia. doi: 10.1038/leu.2016.368

48. de Rooij MFM, Kuil A, Geest CR, Eldering E, Chang BY, Buggy JJ, Pals ST, Spaargaren M (2012) The clinically active BTK inhibitor PCl-32765 targets B-cell receptor- and chemokine-controlled adhesion and migration in chronic lymphocytic leukemia. Blood 119:2590-2594

49. Herman SEM, Mustafa RZ, Jones J, Wong DH, Farooqui M, Wiestner A (2015) Treatment with Ibrutinib Inhibits BTK- and VLA-4-Dependent Adhesion of Chronic Lymphocytic Leukemia Cells In Vivo. Clin Cancer Res Off J Am Assoc Cancer Res 21:4642-4651

50. Prebet T, Lhoumeau A-C, Arnoulet C, et al (2010) The cell polarity PTK7 receptor acts as a modulator of the chemotherapeutic response in acute myeloid leukemia and impairs clinical outcome. Blood 116:2315-2323 
Figure legends

Figure 1. ROR pseudokinases structure and sequence alignment.

A. Schematic diagram of ROR1. The extracellular region comprises of an immunoglobulin (IgG)-like domain, a cysteine-rich domain (CRD) or Frizzled domain and a Kringle domain. The cytoplasmic domain contains the pseudokinase domain followed by two serine-threonine rich domains flanking a proline-rich domain. The CRD is important for binding of Wnt ligands. Kringle domain is required for ROR2 heterodimerization in response to Wnt5a ligand binding, whereas the intracellular proline-rich domain binds cytoplasmic adaptor proteins.

B. Sequence alignment of the human ROR1 and ROR2 pseudokinase domains (amino acids 473-746) by ClustalW-MUSCLE method. Residues mutated in ROR pseudokinase domains are depicted in red color. Consensus sequences of typical kinase domain are depicted in green color and positioned above the corresponding residues in RORs.

Figure 2: Co-targeting ROR1 and BCR pathways in B-ALL and CLL.

Diagram of model for how inhibition of ROR1 signaling works in synergy with inhibition of BCR signaling in B-ALL or CLL models. Targeting ROR1 with cirmtuzumab (magenta color) and BTK with ibrutinib (grey color) in CLL cells results in synergistic inhibition of cell proliferation, survival and cell-migration. In B-ALL cells, inhibition of BTK by dasatinib (navy-blue color) results in upregulation of ROR1 expression, whereas inhibition of both, ROR1 (green color) and BTK leads to simultaneous inhibition of cancer cell biological functions. 


\section{Figure 1}

A

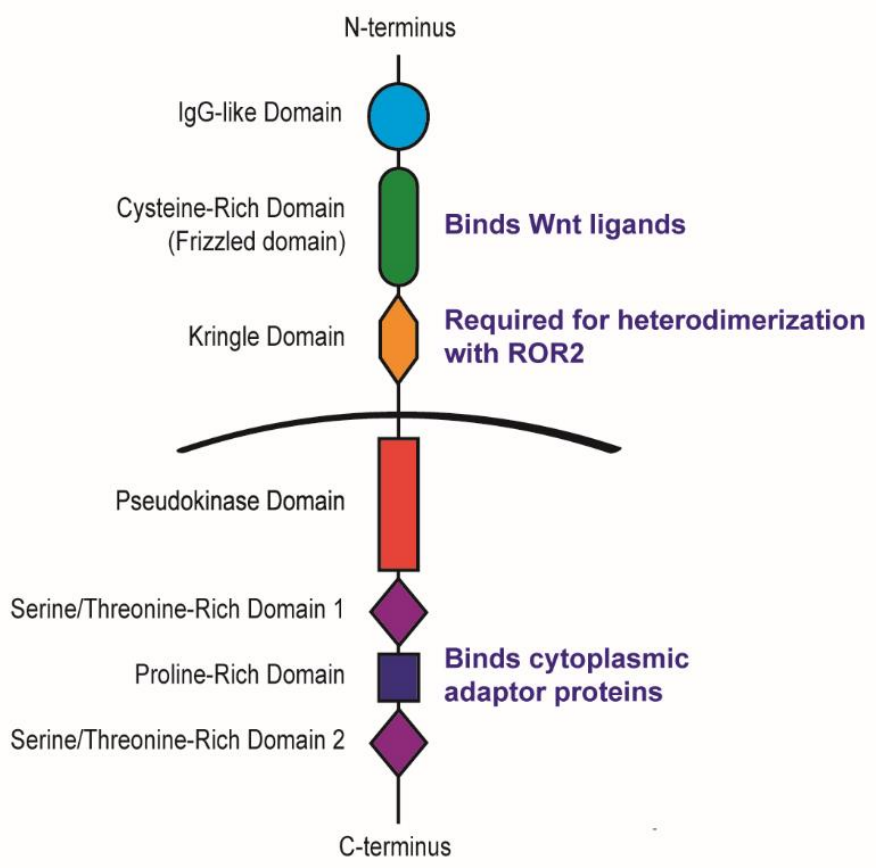

B

GxGxxG (Glycine-rich loop) Invariant lysine

ROR1 VRFMEEIGECAFGKIYKGHLYLPGM-DHAQLVAIKTLKDYNNPQQWTEFQQEASLMAELH

ROR2 VRFMEEIGEDRFGKVYKGHLFGPAPGEQTQAVAIKTLKDKAEGPLREEFRHEAMLRARLQ

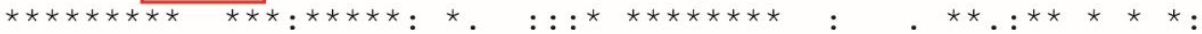

ROR1 HPNIVCLLGAVTQEQPVCMLFEYINQGDLHEFLIMRSPHSDVGCSSDEDGTVKSSLDHGD

ROR2 HPNVVCLLGVVTKDQPLSMIFSYCSHGDLHEFLVMRSPHSDVG-STDDDRTVKSALEPPD

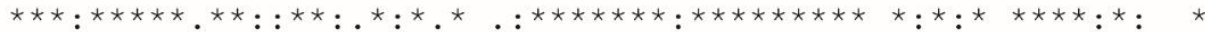

HRD (Catalytic loop) DFG (Mg-binding loop)

ROR1 FLHIAIQIAAGMEYLSSHFFVHKDLAARNILIGEQLHVKISDLGLSREIYSADYYRVQSK

ROR2 FVHLVAQIAAGMEYLSSHHVVHKDLATRNVLVYDKLNVKISDLGLFREVYAADYYKLLGN

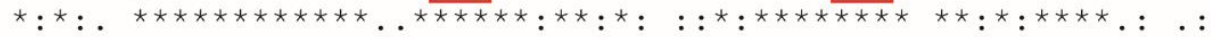

ROR1 SLLPIRWMPPEAIMYGKFSSDSDIWSFGVVLWEIFSFGLQPYYGFSNQEVIEMVRKRQLL ROR2 SLLPIRWMAPEAIMYGKFSIDSDIWSYGVVLWEVFSYGLQPYCGYSNQDVVEMIRNRQVL

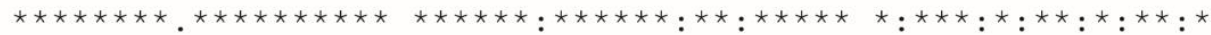

ROR1 PCSEDCPPRMYSLMTECWNEIPSRRPREKDIHVRL

ROR2 PCPDDCPAWVYALMIECWNEFPSRRPREKDIHSRI

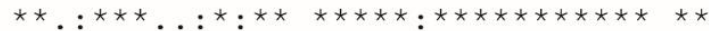


Figure 2

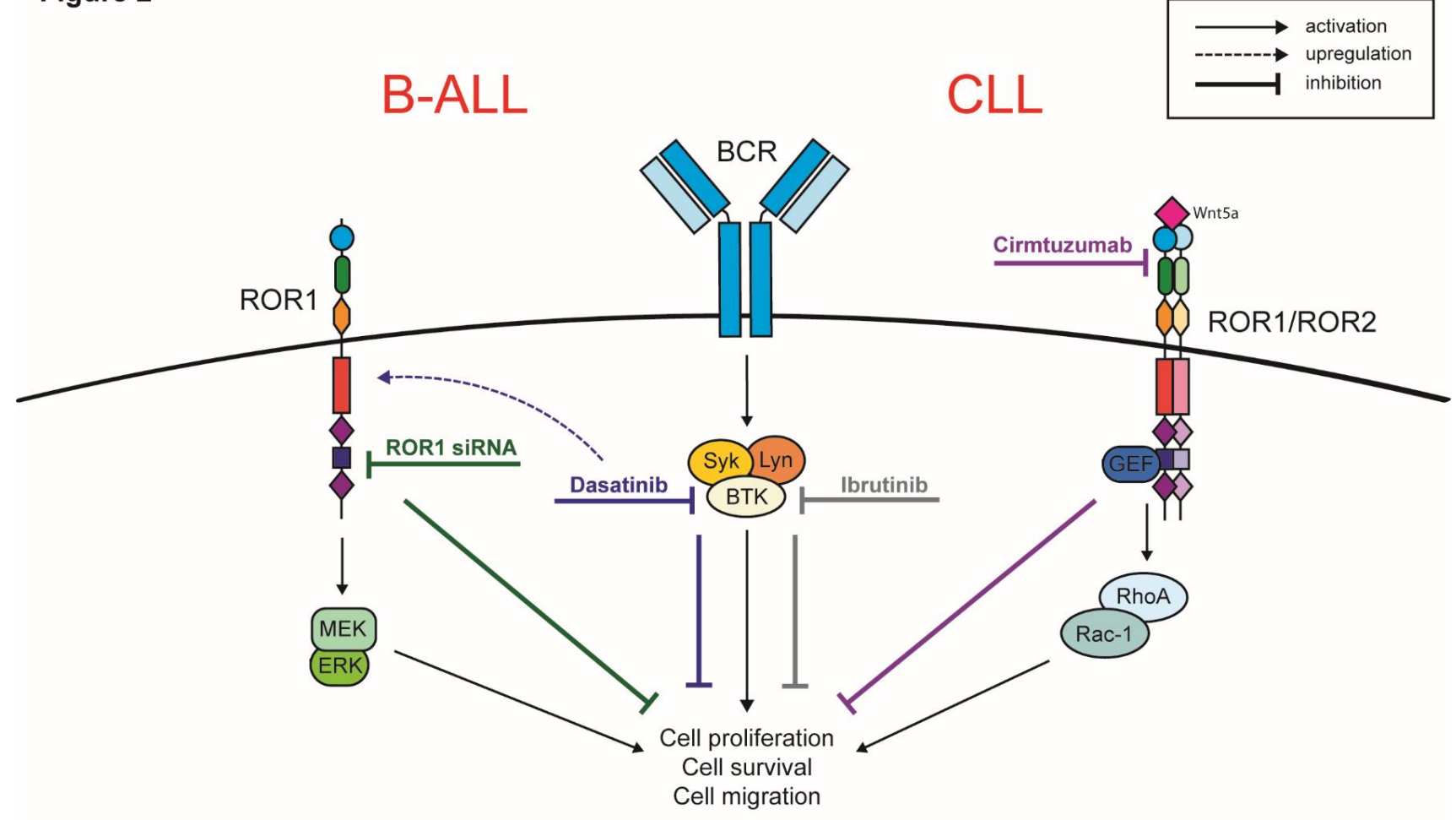

